\title{
SETS OF UNIQUENESS FOR THE VIBRATING STRING PROBLEM
}

BY

\author{
VICTOR L. SHAPIRO( ${ }^{(1)}$
}

1. Introduction. Let $u(x, y)$ be a function in $L^{1}\left[D\left(x_{0}, y_{0}, r_{0}\right)\right]$ where $D\left(x_{0}, y_{0}, r_{0}\right)$ is the open disc with center $\left(x_{0}, y_{0}\right)$ and radius $r_{0}$. We define the upper generalized wave of $u$ at $\left(x_{0}, y_{0}\right)$, designated by $\square^{*} u\left(x_{0}, y_{0}\right)$, to be

$$
\square^{*} u\left(x_{0}, y_{0}\right)=\limsup _{r \rightarrow 0} 24\left(\pi r^{6}\right)^{-1} \int_{D(0,0, r)} u\left(x_{0}+x, y_{0}+y\right)\left(x^{2}-y^{2}\right) d x d y .
$$

In a similar manner using lim inf, we define $\square_{*} u\left(x_{0}, y_{0}\right)$. If $\square^{*} u\left(x_{0}, y_{0}\right)=\square_{*} u\left(x_{0}, y_{0}\right)$, we say the generalized wave of $u$ exists at $\left(x_{0}, y_{0}\right)$, and we designate this common value by $\square u\left(x_{0}, y_{0}\right)$. We note in particular that if $u$ is a bounded Borel function defined in a neighborhood of the point $\left(x_{0}, y_{0}\right)$ and if

$$
\lim _{r \rightarrow 0} 4\left(\pi r^{2}\right)^{-1} \int_{0}^{2 \pi} u\left(x_{0}+r \cos \theta, y_{0}+r \sin \theta\right) \cos 2 \theta d \theta=\alpha,
$$

then $\square u\left(x_{0}, y_{0}\right)$ exists and equals $\alpha$.

(For convenience of notation in this paper, $y$ will play the role that $t$ usually plays in most discussions of the vibrating string problem.)

If $u$ has a second total differential at the point $\left(x_{0}, y_{0}\right)$, i.e., if for $h$ and $k$ tending to zero there exist constants $\alpha_{j}, j=1, \ldots, 5$, such that

$$
u\left(x_{0}+h, y_{0}+k\right)=u\left(x_{0}, y_{0}\right)+\alpha_{1} h+\alpha_{2} k+\frac{1}{2}\left[\alpha_{3} h^{2}+2 \alpha_{4} h k+\alpha_{5} k^{2}\right]+o\left(h^{2}+k^{2}\right),
$$

it is an easy matter to show that $\square u\left(x_{0}, y_{0}\right)$ exists and equals $\alpha_{3}-\alpha_{5}$. Consequently, it follows from Taylor's formula with remainder that if $u$ is in class $C^{2}$ in a neighborhood of the point $\left(x_{0}, y_{0}\right)$, then $\square u\left(x_{0}, y_{0}\right)$ exists and equals $u_{x x}\left(x_{0}, y_{0}\right)-u_{y y}\left(x_{0}, y_{0}\right)$. If $g(x)=x^{n}$, a direct computation shows that

$$
\int_{-\pi}^{\pi} g\left(x_{0}+r \cos \theta+y_{0}+r \sin \theta\right) \cos 2 \theta d \theta=0 \text { for } r>0,
$$

and

$$
\int_{-\pi}^{\pi} g\left(x_{0}+r \cos \theta-y_{0}-r \sin \theta\right) \cos 2 \theta d \theta=0 \text { for } r>0 .
$$

Consequently, if $f(x)$ is a continuous function defined on the real line, then it

Received by the editors September 20, 1968.

(') Research sponsored by the Air Force Office of Scientific Research, Office of Aerospace Research, United States Air Force, under AFOSR Grant No. AF-AFOSR 694-66. 
follows from the Weierstrass approximation theorem that both $\square u(x, y)=0$ and $\square v(x, y)=0$ where $u(x, y)=f(x+y)$ and $v(x, y)=f(x-y)$; so we see that $\square$ is indeed a true generalization for the wave operator usually given in terms of partial derivatives.

(The connection between the usual weak wave operator and the generalized wave operator defined here will be discussed in the appendix of this paper ( $\$ 5)$. It suffices to say at this point that for a function $u$ locally in $L^{1}$ on a disc, the following fact obtains:

If the weak wave of $u=0$ on the disc, then $\square u=0$ on the disc; the converse is false.)

We next define what is meant by a set of uniqueness for the vibrating string problem.

In the sequel, $S$ will designate the infinite half-strip $\{(x, y): 0 \leqq x \leqq \pi, 0 \leqq y<\infty\}$, $S^{\circ}$ will designate its interior, and $\partial S$ its boundary. $S_{1}$ will be the set

$$
\{(x, 0): 0 \leqq x \leqq \pi\}
$$

so in particular $S_{1} \subset \partial S$.

We say a Borel set $Q \subset S_{1}$ is a set of uniqueness for the vibrating string problem:

If whenever $u(x, y)$ meet conditions (i), (ii),

(iii), and (iv), then $u(x, y)$ is identically zero in $S$;

where conditions (i), (ii), (iii), and (iv) are defined as follows:

(i) $u(x, y)$ is continuous in $S$,

(ii) $\square u(x, y)=0$ for $(x, y)$ in $S^{\circ}$,

(iii) $u(x, y)=0$ for $(x, y)$ in $\partial S$,

(iv) $u_{y}(x, y)=0$ for $(x, y)$ in $S_{1}-Q$.

(To be quite explicit, in (iv) we mean $\lim _{y \rightarrow 0+}[u(x, y)-u(x, 0)] / y=0$ for $(x, 0)$ in $\left.S_{1}-Q.\right)$

The main theorem that we intend to prove in this paper is the following:

THEOREM 1. A necessary and sufficient condition that a Borel set $Q \subset S_{1}$ be a set of uniqueness for the vibrating string problem is that $Q$ be countable.

We note that in the above theorem the only differentiability requirement (other than the existence of the generalized wave operator in $S^{\circ}$ ) is the existence of the first derivative with respect to $y$ at the points of $S_{1}-Q$.

2. Proof of the necessary condition of Theorem 1. Suppose we are given an uncountable Borel set $Q \subset S_{1}$. Then $Q$ contains a nonempty, nowhere dense, perfect subset $P\left[3\right.$, p. 205] which is contained in the interior of $S_{1}$ and which we divide into two nonempty, nowhere dense, perfect disjoint subsets $P_{1}$ and $P_{2}$ where the points of $P_{1}$ lie completely to the left of the points of $P_{2}$. (So, in particular we have $P_{1} \cup P_{2}=P \subset Q$.) 
For $j=1,2$, let $P_{j}^{\prime}=\left\{x:(x, 0)\right.$ in $\left.P_{j}\right\}, a_{j}=\inf \left\{x: x\right.$ in $\left.P_{j}^{\prime}\right\}$ and $b_{j}=\sup \left\{x: x\right.$ in $\left.P_{j}^{\prime}\right\}$. Then $0<a_{1}<b_{1}<a_{2}<b_{2}<\pi$, and $P_{1}^{\prime}$ and $P_{2}^{\prime}$ are nonempty perfect sets contained in the interval $\left[a_{1}, b_{1}\right]$ and $\left[a_{2}, b_{2}\right]$, respectively.

Next for $j=1,2$, on the interval $\left[a_{j}, b_{j}\right]$, we construct a Lebesgue-Cantor function $L_{j}(x)$ for the set $P_{j}^{\prime},\left[4\right.$, p. 101]. $L_{j}(x)$ then has the following properties: $L_{j}(x)$ is continuous and monotonically nondecreasing on the interval $\left[a_{j}, b_{j}\right] ; L\left(a_{j}\right)=0$ and $L\left(b_{j}\right)=1 ; L_{j}(x)$ is constant on the open contiguous intervals of $P_{j}^{\prime}$.

We next define $F(x)$ for $-\pi \leqq x \leqq \pi$ as follows:

$$
\begin{aligned}
F(x) & =0 & & 0 \leqq x \leqq a_{1} \\
& =L_{1}(x) & & a_{1} \leqq x \leqq b_{1} \\
& =1 & & b_{1} \leqq x \leqq a_{2} \\
& =1-L_{2}(x) & & a_{2} \leqq x \leqq b_{2} \\
& =0 & & b_{2} \leqq x \leqq \pi \\
& =F(-x) & & -\pi \leqq x \leqq 0 .
\end{aligned}
$$

We define $F(x)$ for $-\infty<x<\infty$ by periodicity of period $2 \pi$ and set $u(x, y)$ $=F(x+y)-F(x-y)$ for $(x, y)$ in $S$. We observe from (2) that $u\left(b_{1} / 2, b_{1} / 2\right)=F\left(b_{1}\right)=1$ and therefore $u(x, y)$ is not identically zero. Consequently the necessary condition of Theorem 1 will be established once we show that $u(x, y)$ meets conditions (i), (ii), (iii), and (iv) above.

That $u$ meets condition (i) follows from the fact that $F(x)$ is a continuous periodic function. That $u$ meets condition (ii) follows from the same fact and one of the remarks made in the introduction (namely that $\square F(x+y)=\square F(x-y)=0$ ).

To show that $u$ satisfies (iii), we observe that $u(0, y)=F(y)-F(-y)=0, u(\pi, y)$ $=F(\pi+y)-F(\pi-y)=F(\pi+y)-F(y-\pi)=0$, and $u(x, 0)=F(x)-F(x)=0$. Therefore $u(x, y)=0$ for $(x, y)$ in $\partial S$ and $u$ meets condition (iii).

We shall show $u$ satisfies (iv) by showing $u_{y}=0$ for $(x, y)$ in $S_{1}-\left(P_{1} \cup P_{2}\right)$. In particular let $\left(x_{0}, 0\right)$ be in $S_{1}-\left(P_{1} \cup P_{2}\right)$. Then it follows from (2) that there exists an $h_{0}>0$ such that $F(x)$ is constant in the interval $\left(x_{0}-h_{0}, x_{0}+h_{0}\right)$. Therefore for $0 \leqq y<h_{0}, F\left(x_{0}+y\right)-F\left(x_{0}-y\right)=0$. We conclude that

$$
\lim _{y \rightarrow 0+}\left[u\left(x_{0}, y\right)-u\left(x_{0}, 0\right)\right] y^{-1}=0 .
$$

Therefore $u$ meets condition (iv), and the proof of the necessary condition of Theorem 1 is complete.

3. Fundamental lemmas. In this section we shall establish some lemmas in double trigonometric series which will be needed to establish the sufficiency condition of the theorem.

We shall use the following notation: $X=(x, y), M=(m, n),(M, X)=m x+n y$, $E_{2}=$ the plane, and $T_{2}=\{X:-\pi<x \leqq \pi,-\pi<y \leqq \pi\} . M$ will always designate an integral lattice point, and for $f$ in $L^{1}\left(T_{2}\right)$, we shall set

$$
\hat{f}(M)=\left(4 \pi^{2}\right)^{-1} \int_{T_{2}} f(X) \exp (-i(M, X)) d X .
$$


Given $f$ in $L^{1}\left(T_{2}\right)$ (and real-valued unless explicitly stated otherwise), we shall say that $f$ is extended by periodicity to all of $E_{2}$ if $f$ is defined in all of $E_{2}$ and is periodic of period $2 \pi$ in each variable.

The first lemma we prove is the following:

LEMMA 1. Let $f(X)$ be in $L^{1}\left(T_{2}\right)$ and extended by periodicity to all of $E_{2}$. Set $W(X, t)=-\sum_{M} \hat{f}(M)\left(m^{2}-n^{2}\right) \exp (i(M, X)-|M| t)$. Then for every $X$ in $E_{2}$, the following inequalities prevail:

(a) $\square_{*} f(X) \leqq \lim \inf _{t \rightarrow 0} W(X, t)$,

(b) $\lim \sup _{t \rightarrow 0} W(X, t) \leqq \square^{*} f(x)$.

To prove the lemma, we clearly need only prove (b), for (a) will follow from (b) when $f$ is replaced by $-f$.

To prove (b), we need only establish it in the special case $X=0$. If $\square^{*} f(0)=+\infty$,

(b) is established. Consequently, we can suppose that $\square^{*} f(0)<+\infty$.

Let

$$
\square^{*} f(0)<p
$$

where $p$ is a finite number. The lemma will be established if we can show

$$
\limsup _{t \rightarrow 0} W(0, t) \leqq p
$$

To show that (4) holds, we observe on differentiating under the integral sign in $[5$, formula 17, p. 56] (which we may clearly do) that

where

$$
\begin{aligned}
W(0, t) & =15 t(2 \pi)^{-1} \int_{E_{2}} f(X)\left(x^{2}-y^{2}\right)\left[t^{2}+|X|^{2}\right]^{-7 / 2} d X \\
& =15 t(2 \pi)^{-1} \int_{0}^{\infty}\left[t^{2}+r^{2}\right]^{-7 / 2} d A(r)
\end{aligned}
$$

$$
A(r)=\int_{D(0, r)} f(X)\left(x^{2}-y^{2}\right) d X
$$

Since $A(r)=O\left(r^{4}\right)$ as $r \rightarrow \infty$, it follows from (5) that

$$
W(0, t)=105 t(2 \pi)^{-1} \int_{0}^{\infty} r A(r)\left[t^{2}+r^{2}\right]^{-9 / 2} d r .
$$

Using (3) and (6), we next choose $r_{0}$ such that

$$
A(r)<p r^{6} \pi(24)^{-1} \text { for } 0<r<r_{0} .
$$

It consequently follows from (7), (8), and the fact that $A(r)=O\left(r^{4}\right)$ as $r \rightarrow \infty$ that

$$
W(0, t) \leqq 35 p(16)^{-1} t \int_{0}^{r_{0}} r^{7}\left[t^{2}+r^{2}\right]^{-9 / 2} d r+o(1)
$$


We infer from this last fact that

$$
\limsup _{t \rightarrow 0} W(0, t) \leqq 35 p(16)^{-1} \int_{0}^{\infty} r^{7}\left[1+r^{2}\right]^{-9 / 2} d r .
$$

Since $\int_{0}^{\infty} r^{7}\left[1+r^{2}\right]^{-9 / 2} d r=16 / 35$, (4) follow from (9), and the proof to the lemma is complete.

For $f$ in $L^{1}\left[D\left(X_{0}, h_{0}\right)\right]$ we shall set

$$
f_{h}\left(X_{0}\right)=\left(\pi h^{2}\right)^{-1} \int_{D\left(X_{0}, h\right)} f(X) d X \text { for } 0<h<h_{0} .
$$

Also, $\Sigma^{\prime}$ will designate a summation over the integral lattice points in the plane with the 0-lattice point excluded, and $\bar{E}$ will designate the closure of the set $E$.

The next lemma we prove is the following:

LEMMA 2. Let $f(X)$ be in $L^{\infty}\left(T_{2}\right)$ and extended by periodicity to all of $E_{2}$. Let $f(X)$ be a function in $L^{2}\left(T_{2}\right)$ extended by periodocity to all of $E_{2}$ having

$$
-\sum^{\prime} \hat{f}(M)\left(m^{2}-n^{2}\right)|M|^{-2} \exp i(M, X)
$$

as its Fourier series. Suppose that

(i) $f(X)$ is continuous in the disc $D\left(X_{0}, 4 h_{0}\right), 0<h_{0}<1$, and

(ii) $\square^{*} f(X)$ and $\square * f(X)$ are finite valued in $D\left(X_{0}, 4 h_{0}\right)$.

Then

$$
\lim _{h \rightarrow 0}\left[\sup _{X \text { in } \bar{D}\left(X_{0}, h_{0}\right) \text { and }|P| \leqq h}\left|\tilde{f}_{h}(X+P)-\tilde{f}_{h}(X)\right|\right]=0 .
$$

To prove the lemma, we first set

$$
K(x)=(4 \pi)\left(x^{2}-y^{2}\right) /|X|^{4} \quad X \neq 0,
$$

and for $X / 2 \pi$ not equal to an integral lattice point we set

$$
K^{*}(X)=K(X)+\lim _{R \rightarrow \infty} \sum_{1 \leqq|M| \leqq R}[K(X+2 \pi M)-K(2 \pi M)] .
$$

Now $K^{*}(X)$ is a continuous periodic function in $E_{2}-\bigcup_{M}\{2 \pi M\}$ (see [2, pp. 251-252]) with the series in (11) converging uniformly for $X$ in $T_{2}$. We set

$$
{ }_{\varepsilon} f(X)=(2 \pi)^{-2} \int_{T_{2}-D(0, \varepsilon)} f(X-P) K^{*}(P) d P,
$$

and observe that ${ }_{\varepsilon} f(X)$ is a continuous periodic function in $E_{2}$.

It follows from condition (ii) in the hypothesis of the lemma that

$$
\lim _{\varepsilon \rightarrow 0} \int_{D(0,1)-D(0, \varepsilon)} f(X-P) K(P) d P
$$

exists and is finite for $X$ in $D\left(X_{0}, 4 h_{0}\right)$. 
From [2, pp. 253-261] we obtain further

$$
\lim _{\varepsilon \rightarrow 0} \tilde{f}(X)=\tilde{f}(X) \quad \text { almost everywhere in } E_{2},
$$

and in particular from (13), we have that

$$
\lim _{\varepsilon \rightarrow 0} \tilde{f}(X)=\tilde{f}(X) \text { is finite in } D\left(X_{0}, 4 h_{0}\right) \text {. }
$$

Also, it follows from [2, pp. 253-254] that $\tilde{f}(X)$ is in $L^{2}\left(T_{2}\right)$ and periodic in $E_{2}$ and furthermore

$$
\lim _{\varepsilon \rightarrow 0} \int_{T_{2}}\left|{ }_{\varepsilon} \tilde{f}(X)-\tilde{f}(X)\right|^{2} d x=0 .
$$

Observing that $\lim _{\varepsilon \rightarrow 0} \int_{T_{2}-D(0, \varepsilon)} K^{*}(P) d P=0$, we conclude from (12) and (16) that

$$
f_{h}(X)=\lim _{\varepsilon \rightarrow 0}(2 \pi)^{-2} \int_{T_{2}-D(0, \varepsilon)}\left[f_{h}(X-P)-f_{h}(X)\right] K^{*}(P) d P .
$$

Now it is an easy matter to see that since $f$ is a periodic function in $L^{\infty}\left(T_{2}\right)$ that for each $h>0$ there is a constant $c_{h}$ such that

$$
\left|f_{h}(X)-f_{h}\left(X^{\prime}\right)\right| \leqq c_{h}\left|X-X^{\prime}\right| \text { for } X \text { and } X^{\prime} \text { in } E_{2} \text {. }
$$

In particular since $K^{*}(P)$ has a singularity of the form $O\left(|P|^{-2}\right)$ in a neighborhood of the origin, it follows from (18) that for fixed $X,\left[f_{h}(X-P)-f_{h}(X)\right] K^{*}(P)$ is in $L^{1}$ on $T_{2}$ as a function of $P$. Consequently, we can write (17) in the form

$$
\tilde{f}_{h}(X)=(2 \pi)^{-2} \int_{T_{2}}\left[f_{h}(X-P)-f_{h}(X)\right] K^{*}(P) d P .
$$

But then

$$
\tilde{f}_{h}\left(X+P^{\prime}\right)=(2 \pi)^{-2} \int_{T_{2}}\left[f_{h}\left(X+P^{\prime}-P\right)-f_{h}\left(X+P^{\prime}\right)\right] K^{*}(P) d P,
$$

and therefore

$$
\tilde{f}_{h}\left(X+P^{\prime}\right)=(2 \pi)^{-2} \int_{T_{2}}\left[f_{h}(X-P)-f_{h}\left(X+P^{\prime}\right)\right] K^{*}\left(P+P^{\prime}\right) d P .
$$

We designate the modulus of continuity of $f$ in $\bar{D}\left(X_{0}, 2 h_{0}\right)$ by $\omega$. Thus

$$
\omega(s)=\sup _{X \text { and } X^{\prime} \text { in } \bar{D}\left(X_{0}, 2 h_{0}\right) \text { and }\left|X-X^{\prime}\right| \leqq s}\left|f(X)-f\left(X^{\prime}\right)\right| .
$$

Now for $X$ in $\bar{D}\left(X_{0}, h_{0}\right)$ and $0<h<h_{0} / 100$

$$
\begin{aligned}
\int_{D(0,2 h)} & \left|\left[f_{h}(X-P)-f_{h}(X)\right]\right|\left|K^{*}(P)\right| d P \\
\leqq & \int_{D(0,2 h)-D(0, h / 2)} \omega(2 h)\left|K^{*}(P)\right| d P \\
& \quad+\int_{D(0, h / 2)}\left|K^{*}(P)\right|\left(\pi h^{2}\right)^{-1}\left|\int_{D(0, h)}\left[f\left(X+P^{\prime \prime}-P\right)-f\left(X+P^{\prime \prime}\right)\right] d P^{\prime \prime}\right| d P .
\end{aligned}
$$


Now using circular lunes, we see that for $|P|<h / 2$,

$$
\begin{aligned}
\left|\int_{D(0, h)}\left[f\left(X+P^{\prime \prime}-P\right)-f\left(X+P^{\prime \prime}\right)\right] d P^{\prime \prime}\right| & \leqq \omega(3 h) \int_{D(0,|P|+h)-D(0, h)} d P^{\prime \prime} \\
& \leqq \omega(3 h) 3|P| h .
\end{aligned}
$$

(To see that the estimate in (23) is actually correct, we observe that the integral there is $\int_{Q} f\left(X+P^{\prime \prime}\right) d P^{\prime \prime}-\int_{Q^{\prime}} f\left(X+P^{\prime \prime}\right) d P^{\prime \prime}$, where $Q$ and $Q^{\prime}$ are congruent circular lunes with the total diameter of $Q \cup Q^{\prime}$ being smaller than $5 h / 2$ and $Q$ being contained in the annulus of inner radius $h$ and outer radius $h+|P|$.)

Observing that for $P$ in $T_{2},\left|K^{*}(P)\right|$ is majorized by a constant multiple of $|P|^{-2}$, we conclude from (22) and (23) that for $X$ in $\bar{D}\left(X_{0}, h_{0}\right)$

$$
\int_{D(0,2 h)}\left|f_{h}(X-P)-f_{h}(X)\right|\left|K^{*}(P)\right| d P \leqq c_{1} \omega(3 h)
$$

where $c_{1}$ is a constant independent of $X$ and $h$.

Likewise with $\left|P^{\prime}\right| \leqq h$ and $X$ in $\bar{D}\left(X_{0}, h_{0}\right)$,

$$
\begin{aligned}
\int_{D(0,2 h)} & \left|f_{h}(X-P)-f_{h}\left(X+P^{\prime}\right)\right|\left|K^{*}\left(P+P^{\prime}\right)\right| d P \\
& \leqq \int_{D(0,3 h)}\left|f_{h}\left(X+P^{\prime}-P\right)-f_{h}\left(X+P^{\prime}\right)\right|\left|K^{*}(P)\right| d P \\
& \leqq c_{2} \omega(3 h)
\end{aligned}
$$

where $c_{2}$ is a constant independent of $X$ and $h$.

We therefore obtain from (19), (20), (24), and this last computation that for $X$ in $\bar{D}\left(X_{0}, h_{0}\right),\left|P^{\prime}\right| \leqq h$, and $0<h<h_{0} / 100$,

$$
\begin{aligned}
& \left|\tilde{f}_{h}\left(X+P^{\prime}\right)-\tilde{f}_{h}(X)\right| \\
& \leqq c_{3} \omega(3 h)+\mid(2 \pi)^{-2} \int_{T_{2}-D(0,2 h)}\left[f_{h}(X-P)-f_{h}(X)\right] \\
& \cdot\left[K^{*}\left(P+P^{\prime}\right)-K^{*}(P)\right] d P \\
& +(2 \pi)^{-2}\left|f_{h}(X)-f_{h}\left(X+P^{\prime}\right)\right|\left|\int_{T_{2}-D(0,2 h)} K^{*}\left(P+P^{\prime}\right) d P\right| \text {, }
\end{aligned}
$$

where $c_{3}$ is a constant independent of $X$ and $h$.

Setting

$$
T_{2}^{1 / 8}=\left\{P^{\prime \prime}: P^{m}+P^{\prime \prime \prime \prime}, P^{m} \text { in } T_{2}, \text { and }\left|P^{\prime \prime \prime \prime}\right| \leqq 8^{-1}\right\},
$$

we see from (11) that

(26) $K^{*}(P)=K(P)+K^{\prime}(P)$ for $P$ in $T_{2}^{1 / 8}$ where $K^{\prime}(P)$ is continuous in $T_{2}^{1 / 8}$.

Consequently,

$$
\left|\int_{T_{2}-D(0,2 h)} K^{*}\left(P+P^{\prime}\right) d P\right| \leqq c_{4}+\left|\int_{D(0,1)-D\left(P^{\prime}, 2 h\right)} K(P) d P\right|,
$$

where $c_{4}$ is a constant independent of $P^{\prime}$ and $h$. 
Now it follows from (10) and the fact that $\left|P^{\prime}\right| \leqq h$ that

$$
\begin{aligned}
\left|\int_{D(0,1)-D\left(P^{\prime}, 2 h\right)} K(P) d P\right| & \leqq 4 \pi \int_{D\left(0,\left|P^{\prime}\right|+2 h\right)-D\left(P^{\prime}, 2 h\right)}|P|^{-2} d P \\
& \leqq 8 \pi^{2} \int_{h}^{3 h} r^{-1} d r \leqq 16 \pi^{2} .
\end{aligned}
$$

We consequently conclude from (25), (27), and (28) that for $X$ in $\bar{D}\left(X_{0}, h_{0}\right)$, $\left|P^{\prime}\right| \leqq h$, and $0<h<h_{0} / 100$

$$
\begin{aligned}
& \left|\tilde{f}_{h}\left(X+P^{\prime}\right)-\tilde{f}_{h}(X)\right| \\
& \leqq c_{5} \omega(3 h)+\mid(2 \pi)^{-2} \int_{T_{2}-D(0,2 h)}\left[f_{h}(X-P)-f_{h}(X)\right] \\
& \cdot\left[K^{*}\left(P+P^{\prime}\right)-K^{*}(P)\right] d P
\end{aligned}
$$

where $c_{5}$ is a constant independent of $X$ and $h$.

Next, we set for $0<h<h_{0} / 100$

$$
\omega_{1}^{*}(h)=\sup _{\left|P^{\prime}\right| \leqq h} \int_{T_{2}-D\left(0, h_{0} / 2\right)}\left|K^{*}\left(P+P^{\prime}\right)-K^{*}(P)\right| d P
$$

and obtain from the fact that $f$ is in $L^{\infty}\left(T_{2}\right)$ and from (29) that the last expression on the right-hand side of the inequality in (29) is majorized by

$$
c_{6} \omega_{1}^{*}(h)+\left|(2 \pi)^{-2} \int_{D\left(0, h_{0} / 2\right)-D(0,2 h)}\left[f_{h}(X-P)-f_{h}(X)\right]\left[K^{*}\left(P+P^{\prime}\right)-K^{*}(P)\right] d P\right|
$$

where $c_{6}$ is a constant independent of $X$ and $h$.

Next with $K^{\prime}(P)$ the continuous function in $T_{2}^{1 / 8}$ defined in (26), we set

$$
\omega_{2}^{\prime}(h)=\sup _{\left|X-X^{\prime}\right| \leqq h, X \text { and } X^{\prime} \text { in } T_{2}^{1 / 8}}\left|K^{\prime}(X)-K^{\prime}\left(X^{\prime}\right)\right|,
$$

and we obtain that the second expression in (31) is majorized by

$$
c_{7} \omega_{2}^{\prime}(h)+(2 \pi)^{-2} \int_{D\left(0, h_{0} / 2\right)-D(0,2 h)} \omega(|P|)\left|K\left(P+P^{\prime}\right)-K(P)\right| d P
$$

where $c_{7}$ is a constant independent of $X$ and $h$.

For $2 h \leqq|P|<1 / 2$ and $\left|P^{\prime}\right|<h$, we see from (10) that there is a constant $c_{8}$ independent of $h$ such that $\left|K\left(P+P^{\prime}\right)-K(P)\right| \leqq c_{8} h|P|^{-3}$. We conclude from this fact, (29), (31), and (33) that for $X$ in $\bar{D}\left(X_{0}, h_{0}\right),\left|P^{\prime}\right| \leqq h$, and $0<h<h_{0} / 100$,

$$
\left|f_{h}\left(X+P^{\prime}\right)-f_{h}^{\prime}(X)\right| \leqq c_{9}\left[\omega(3 h)+\omega_{1}^{*}(h)+\omega_{2}^{\prime}(h)+h \int_{2 h}^{h_{0} / 2} \omega(r) r^{-2} d r\right]
$$

where $c_{9}$ is a constant independent of $X$ and $h$. The conclusion to the lemma follows immediately from (21), (30), (32), and (33').

The proof to the above lemma was motivated somewhat by [8, pp. 121-122]. 
The next lemma we prove is the following:

LEMMA 3. Let $f$ and $f$ be two functions in $L^{2}\left(T_{2}\right)$ and extended by periodicity to all of $E_{2}$ with $\tilde{f}$ having $-\sum^{\prime} \hat{f}(M)\left(m^{2}-n^{2}\right)|M|^{-2} e^{i(M, X)}$ as its Fourier series. Let $0<h_{1}$ $<h_{2}$. Then for every $X$,

$$
(\pi)^{-1} \int_{D\left(0, h_{2}\right)-D\left(0, h_{1}\right)} f(X-P)\left(p^{2}-q^{2}\right)|P|^{-4} d P=f_{h_{1}}(X)-f_{h_{2}}(X) .
$$

Since both the right- and left-hand sides of the equality in (34) are continuous periodic functions, to prove the lemma we need only show that they have the same Fourier coefficients.

Setting $g(X)=\tilde{f}_{h_{1}}(X)-\tilde{f}_{h_{2}}(X)$ an easy computation shows that

(35) $\hat{g}(M)=2 \hat{f}(M)\left(m^{2}-n^{2}\right)|M|^{-2}\left[J_{1}\left(|M| h_{2}\right)\left(|M| h_{2}\right)^{-1}-J_{1}\left(|M| h_{1}\right)\left(|M| h_{1}\right)^{-1}\right]$

for $M \neq 0, \hat{g}(0)=0$.

On the other hand, Fubini's theorem and the periodicity of $f$ show that

$$
\int_{T_{2}} d x\left\{\int_{D\left(0, h_{2}\right)-D\left(0, h_{1}\right)} f(X-P)\left(p^{2}-q^{2}\right)|P|^{-4} d P\right\}=0 .
$$

Also, for $|M| \neq 0$ and $\cos 2 \varphi=\left(m^{2}-n^{2}\right)|M|^{-2}$,

$$
\begin{gathered}
\left(4 \pi^{3}\right)^{-1} \int_{T_{2}} \exp (-i(M, X)) d X\left\{\int_{D\left(0, h_{2}\right)-D\left(0, h_{1}\right)} f(X-P)\left(p^{2}-q^{2}\right)|P|^{-4}\right\} d P \\
=(\pi)^{-1} \hat{f}(M) \int_{h_{1}}^{h_{2}} r^{-1} d r \int_{0}^{2 \pi} \exp (-i|M| r \cos (\theta-\varphi)) \cos 2 \theta d \theta \\
=\left.2\left(m^{2}-n^{2}\right)|M|^{-2} \hat{f}(M) J_{1}(r) r^{-1}\right|_{\substack{|M| h_{1} \\
|M| h_{2}}}
\end{gathered}
$$

(34) and consequently the lemma follows from this last computation, (35), and (36).

Following the notation of [5, Lemma 8, p. 72], we next set

$$
H_{2}(X, t)=\sum^{\prime} i n|M|^{-2} \exp (i(M, X)-|M| t) \text { for } t>0,
$$

and $H_{2}(X)=\lim _{t \rightarrow 0} H_{2}(X, t)$, where this last limit exists for $X \neq 2 \pi M$.

We observe also from [5, Lemma 8, p. 72] that $H_{2}(X)$ is in $L^{1}\left(T_{2}\right)$ and that

$$
\hat{H}_{2}(M)=\operatorname{in}|M|^{-2} \quad M \neq 0 \quad \hat{H}_{2}(0)=0 .
$$

The next lemma we prove is the following:

LEMMA 4. Let $f(X)$ be a function in $L^{\infty}\left(T_{2}\right)$ and extended by periodicity to all of $E_{2}$, and set

$$
f_{2}(X)=\left(4 \pi^{2}\right)^{-1} \int_{T_{2}} f(X-P) H_{2}(P) d P .
$$

Suppose $f(X)$ is continuous in the strip $\left\{(x, y):-\infty<x<\infty\right.$ and $y_{0}-k_{0} \leqq y \leqq y_{0}+k_{0}$ where $\left.k_{0}>0\right\}$. Then $f_{2}\left(x, y_{0}\right)$ is uniformly smooth in $x$. 
We recall $[8, \mathrm{p} .43]$ that $f_{2}\left(x, y_{0}\right)$ (which is a continuous periodic function of $x$ ) is uniformly smooth means

$$
\lim _{h \rightarrow 0+} \sup _{-\pi \leqq x \leqq \pi}\left|f_{2}\left(x+h, y_{0}\right)+f_{2}\left(x-h, y_{0}\right)-2 f_{2}\left(x, y_{0}\right)\right| h^{-1}=0 .
$$

In the sequel, $h$ will always be such that $0<h<1 / 16$. Then

$$
\begin{aligned}
& \left(4 \pi^{2}\right)\left[f_{2}\left(x+h, y_{0}\right)+f_{2}\left(x-h, y_{0}\right)-2 f_{2}\left(x, y_{0}\right)\right] \\
& \quad=\int_{T_{2}}\left[f\left(x-p, y_{0}-q\right)-f\left(x, y_{0}\right)\right]\left[H_{2}(p+h, q)+H_{2}(p-h, q)-2 H_{2}(p, q)\right] d p d q .
\end{aligned}
$$

For $X$ in $T_{2}^{1 / 8}-\{0\}$, let us set

$$
\psi_{2}(X)=H_{2}(X)+(2 \pi) y|X|^{-2} .
$$

By [5, Lemma 8, p. 72], $\psi_{2}(X)$ is harmonic in a domain containing the closure of $T_{2}^{1 / 8}$. Consequently there exists a constant $c_{1}$ such that for $0<h<1 / 16$ and $P$ in $T_{2}$,

$$
\left|\psi_{2}(p+h, q)+\psi_{2}(p-h, q)-2 \psi_{2}(p, q)\right| \leqq c_{1} h^{2} .
$$

We consequently conclude from (38), (39), and (40) that (37) will follow once we show

$$
\lim _{h \rightarrow 0+} h^{-1} \sup _{-\pi \leqq x \leqq \pi}\left|\int_{T_{2}}\left[f\left(x-p, y_{0}-q\right)-f\left(x, y_{0}\right)\right] g_{2}(P, h) d P\right|=0
$$

where

$$
g_{2}(P, h)=q\left\{\left[(h+p)^{2}+q^{2}\right]^{-1}+\left[(h-p)^{2}+q^{2}\right]^{-1}-2|P|^{-2}\right\} .
$$

Next using the fact that $f(x, y)$ is uniformly continuous in the infinite strip $\left\{(x, y):-\infty<x<\infty\right.$ and $\left.y_{0}-k_{0} \leqq y \leqq y_{0}+k_{0}\right\}$ and given $\varepsilon>0$ we choose $\delta$ such that $0<\delta<\min \left(1, k_{0}\right)$ and such that

$$
\left|f\left(x-p, y_{0}-q\right)-f\left(x, y_{0}\right)\right| \leqq \varepsilon \text { for }|P|<\delta .
$$

An easy computation shows that for $P$ in $T_{2}-D(0, \delta)$ and $0<h<\delta / 2,\left|g_{2}(P, h)\right|$ is majorized by a constant multiple of $h^{2}$. Consequently, we conclude from (42) and (43) that

$$
\begin{gathered}
\limsup _{h \rightarrow 0+} h^{-1} \sup _{-\pi \leqq x \leqq \pi}\left|\int_{T_{2}}\left[f\left(x-p, y_{0}-q\right)-f\left(x, y_{0}\right)\right] g_{2}(P, h) d P\right| \\
\leqq \varepsilon \limsup _{h \rightarrow 0+} h^{-1} \int_{D(0 . \delta)}\left|g_{2}(P, h)\right| d P .
\end{gathered}
$$

Now for $0<h<\delta / 2$,

$$
\int_{D(0,2 h)}\left|g_{2}(P, h)\right| d P \leqq 4 \int_{D(0,3 h)}|P|^{-1} d P \leqq 24 \pi h
$$


On the other hand,

$$
\left|g_{2}(P, h)\right| \leqq 96 h^{2}|P|^{-3} \text { for } 2 h \leqq|P| \leqq \delta .
$$

Therefore,

$$
\int_{D(0, \delta)-D(0,2 h)}\left|g_{2}(P, h)\right| d P \leqq 96 \pi h .
$$

We conclude from (45) and (46) that the left side of the inequality in (44) is majorized by $120 \pi \varepsilon$. (41) follows immediately from this, and the proof to the lemma is complete.

The next lemma we establish is the following:

Lemma 5. Let $F(X)$ be in $L^{1}\left(T_{2}\right)$ and extended by periodicity to all of $E_{2}$. For $t>0$, set $W(X, t)=-\sum \hat{F}(M)|M|^{2} \exp (i(M, X)-|M| t)$. Suppose that

(i) $F(X)$ is continuous in $D\left(X_{0}, h_{0}\right), 0<h_{0}<1$,

(ii) $\lim \sup _{t \rightarrow 0}|W(X, t)|<\infty$ in $D\left(X_{0}, h_{0}\right)$,

(iii) $\lim _{t \rightarrow 0} W(X, t)=0$ almost everywhere in $D\left(X_{0}, h_{0}\right)$.

Then $F(X)$ is harmonic in $D\left(X_{0}, h_{0}\right)$.

To prove the lemma, we set

$$
F(X, t)=\sum_{M} \hat{F}(M) \exp (i(M, X)-|M| t) \text { for } t>0 .
$$

By [6, Lemma 6],

$$
\lim _{t \rightarrow 0} \int_{D\left(X_{0}, h\right)}|W(X, t)| d t=0 \text { for } 0<h<h_{0} .
$$

Let $\lambda(X)$ be a function in class $C^{(\infty)}$ which vanishes outside a compact subset of $D\left(X_{0}, h_{0}\right)$. Then since $\Delta F(X, t)=W(X, t)$ (where $\Delta$ as usual designates the Laplace operator) it follows that

$$
\int_{D\left(X_{0}, h_{0}\right)} F(X, t) \Delta \lambda(X) d X=\int_{\left(D x_{0}, h_{0}\right)} W(X, t) \lambda(X) d X .
$$

Consequently, we obtain from this fact and (47) that

$$
\lim _{t \rightarrow 0} \int_{D\left(X_{0}, h_{0}\right)} F(X, t) \Delta \lambda(X) d X=0 .
$$

However, as is well known, $F(X, t) \rightarrow F(X)$ as $t \rightarrow 0$ uniformly on compact subsets of $D\left(X_{0}, h_{0}\right)$ (see [5, Formula (17), p. 56]). We conclude from (48) that

$$
\int_{D\left(X_{0}, h_{0}\right)} F(X) \Delta \lambda(X) d X=0 .
$$

But the continuity of $F(X)$ in $D\left(X_{0}, h_{0}\right)$ plus the fact that (49) holds for every $\lambda$ in class $C^{(\infty)}$ vanishing outside of a compact subset of $D\left(X_{0}, h_{0}\right)$ implies, as is well known, that $F(X)$ is harmonic in $D\left(X_{0}, h_{0}\right)$, and the proof to the lemma is complete. 
The next lemma we establish is the following:

Lemma 6. Let $F(X)$ be in $L^{1}\left(T_{2}\right)$ and extended by periodicity to all of $E_{2}$. For $t>0$, set $W^{\prime}(X, t)=-\sum \hat{F}(M)|M|^{2} \exp \left(i(M, X)-|M|^{2} t\right)$. Suppose $F(X)$ is harmonic in $D\left(X_{0}, h_{0}\right)$ where $0<h_{0}<1$. Then $W^{\prime}(X, t) \rightarrow 0$ as $t \rightarrow 0$ uniformly in $D\left(X_{0}, h_{1}\right)$ where $0<h_{1}<h_{0}$.

The proof is essentially the same as [6, Lemma 5]. We need only observe that the function $S(X, t)=\sum \exp \left(i(M, X)-|M|^{2} t\right)$ is such that $\Delta S(X, t) \rightarrow 0$ uniformly for $X$ in $T_{2}-D\left(0, h_{1}\right)$. (This fact follows immediately from the easily established identity $S(X, t)=(\pi t)^{-1} \sum_{M} \exp \left(-|X+2 \pi M|^{2} / 4 t\right)$.)

4. Proof of the sufficiency condition of Theorem 1. To establish the sufficiency condition of Theorem 1 , it is clear that we need only show $u(x, y)=0$ for $0 \leqq x \leqq \pi$ and $0 \leqq y<\pi$. This fact will follow from the following more general theorem which we shall prove:

THEOREM 2. Let $S^{\pi}$ designate the rectangle $\{(x, y): 0 \leqq x \leqq \pi, 0 \leqq y<\pi\}$ and $S^{\pi \circ}$ represents its interior. Also, let $Q$ be a countable set contained in $S_{1}=\{(x, 0): 0 \leqq x<\pi\}$. Suppose that

(i) $u(x, y)$ is continuous and bounded in $S^{\pi}$;

(ii) $\square^{*} u(X)$ and $\square * u(X)$ are finite for $X$ in $S^{\pi \circ}$;

(iii) $\square u(X)=0$ almost everywhere in $S^{\pi \circ}$;

(iv) $u(x, 0)=0$ for $X$ in $S_{1}$ and $u(0, y)=u(\pi, y)=0$ for $0 \leqq y<\pi$;

(v) $u_{y}(x, 0)=0$ for $X$ in $S_{1}-Q$.

Then $u(X)$ is identically zero for $X$ in $S^{\pi}$.

To prove the theorem, we set

$$
\begin{aligned}
f(X) & =u(X) & & \text { for }(x, y) \text { in } S^{\pi}, \\
& =-u(X) & & \text { for }(-x, y) \text { in } S^{\pi} \\
& =-u(X) & & \text { for }(x,-y) \text { in } S^{\pi} \\
& =u(X) & & \text { for }(-x,-y) \text { in } S^{\pi} \\
& =0 & & \text { if } x= \pm \pi \text { or } y= \pm \pi .
\end{aligned}
$$

We then extend $f$ by periodicity to all of $E_{2}$ by making $f$ periodic of period $2 \pi$ in each variable and observe from the conditions in the hypothesis of the theorem that

$$
f(X) \text { is in } L^{\infty}\left(T_{2}\right)
$$

and that

$$
\begin{aligned}
& \square * f(X) \text { and } \square_{*} f(X) \text { are finite in } E_{2}, \\
& \text { and } \square f(X)=0 \text { almost everywhere in } E_{2} .
\end{aligned}
$$


We also observe that with $\mathscr{S}$ the open strip defined by

$$
\mathscr{S}=\{(x, y):-\infty<x<\infty,-\pi<y<\pi\}
$$

that

$$
f(X) \text { is continuous in } \mathscr{S} \text {. }
$$

Next, we observe from (51) that in particular $f$ is in $L^{2}\left(T_{2}\right)$ and consequently

$$
-\sum^{\prime} \hat{f}(M)\left(m^{2}-n^{2}\right)|M|^{-2} \exp (i(M, X))
$$

is the Fourier series of a function in $L^{2}\left(T_{2}\right)$ which we shall call $\tilde{f}$. From (34) in Lemma 3 and from (1) and (52) we observe that for each fixed $X$ in $E_{2}, f_{h}(X)$ satisfies the Cauchy criterion as $h \rightarrow 0$. Consequently we define $\tilde{f}(X)$ everywhere in $E_{2}$ by

$$
\tilde{f}(X)=\lim _{h \rightarrow 0} \tilde{f}_{h}(X) \text { for } X \text { in } E_{2} \text {. }
$$

We next propose to show with $\mathscr{S}$ given by (53) that

$$
f(X) \text { is continuous in } \mathscr{S} \text {. }
$$

Let $Z$ be the set of discontinuity points of $f(X)$ in $\mathscr{S}$. We propose to show that $Z$ is empty.

First we set

$$
A(r, X)=r^{-6} \int_{D(0, r)} f(X-P)\left(p^{2}-q^{2}\right) d P .
$$

Using the fact that $A(r, X)$ is periodic of period $2 \pi$ in the $x$ and $y$-variables and is jointly continuous in $r$ and $X$ for $r>0$ and $X$ in $E_{2}$, we choose a decreasing sequence $r_{1}>r_{2}>\cdots \rightarrow 0$ such that

$$
\left|A(r, X)-A\left(r_{k}, X\right)\right| \leqq 1 \text { for } X \text { in } E_{2} \text { and } r_{k} \geqq r \geqq r_{k+1} \text {. }
$$

We first propose to show

$$
Z \text { is nowhere dense in } \mathscr{S} \text {. }
$$

Let $\bar{D}\left(X_{0}, h_{0}\right) \subset \mathscr{S}$ where $h_{0}>0$. Then $\left\{A\left(r_{k}, X\right)\right\}_{k=1}^{\infty}$ is a sequence of continuous functions defined on $\bar{D}\left(X_{0}, h_{0}\right)$. Also, it follows from (52) and (58) that

$$
\limsup _{k \rightarrow \infty}\left|A\left(r_{k}, X\right)\right|<\infty \text { for } X \text { in } \bar{D}\left(X_{0}, h_{0}\right) \text {. }
$$

Consequently, there exists (by the Baire category theorem, see [8, p. 29 (12.3i)]) a constant $c_{1}$ and $\bar{D}\left(X_{1}, h_{1}\right) \subset \bar{D}\left(X_{0}, h_{0}\right)$ with $h_{1}>0$ such that $\left|A\left(r_{k}, X\right)\right| \leqq c_{1}$ for $X$ in $\bar{D}\left(X_{1}, h_{1}\right)$ and $k=1,2, \ldots$ But then it follows from (59) that

$$
|A(r, X)| \leqq c_{1}+1 \text { for } X \text { in } \bar{D}\left(X_{1}, h_{1}\right) \text { and } 0<r<r_{1} \text {. }
$$

Next using Lemma 3 we observe that for $0<h<h^{\prime}$

$$
\tilde{f}_{h}(X)-\tilde{f}_{h^{\prime}}(X)=\pi^{-1}\left\{\left.r^{2} A(r, X)\right|_{h} ^{h^{\prime}}+4 \int_{h}^{h^{\prime}} r A(r, X) d r\right\}
$$


We conclude from (61) and (62) that $\lim _{h \rightarrow 0} \tilde{f}_{h}(X)=\tilde{f}(X)$ uniformly for $X$ in $\bar{D}\left(X_{1}, h_{1}\right)$. Consequently, $(60)$ is established.

Next, we observe that

$$
\begin{aligned}
& \tilde{f}(X) \text { continuous in } D\left(X_{0}, h_{0}\right), h_{0}>0, \\
& \text { implies that } \tilde{f}(X) \text { is harmonic in } D\left(X_{0}, h_{0}\right) .
\end{aligned}
$$

To establish (63), we define $W(X, t)$ as in Lemma 1 and recall that Fourier series of $\tilde{f}(X)$ is given by (55). It follows from Lemma 1 and (52) that

$$
\limsup _{t \rightarrow 0}|W(X, t)|<\infty
$$

for every $X$ in $E_{2}$ and $\lim _{t \rightarrow 0} W(X, t)=0$ almost everywhere in $E_{2}$. But then (63) follows from Lemma 5 when we identify $\tilde{f}$ with $-F$ in that lemma.

Next with $\bar{Z}$ designating the closure of $Z$ and $A\left(r_{k}, X\right)$ as in (59), we obtain once again from the Baire category theorem, (59), and the fact that lim $\sup \left|A\left(r_{k}, X\right)\right|$ $<\infty$ for $X$ in $\bar{Z}$ that if $\bar{Z}$ is nonempty, then there exist a constant $c_{2}$ and $D\left(X_{2}, h_{2}\right)$ with $h_{2}>0$ such that

$$
D\left(X_{2}, h_{2}\right) \cap \bar{Z} \neq 0
$$

and

$$
|A(r, X)| \leqq c_{2} \text { for } X \text { in } D\left(X_{2}, 2 h_{2}\right) \cap \bar{Z} \text { and } 0<r<r_{1} .
$$

It follows from (62), (64), and (65) that if $\bar{Z}$ is nonempty, then

$$
\lim _{h \rightarrow 0} \tilde{f}_{h}(X)=\tilde{f}(X) \text { uniformly for } X \text { in } \bar{D}\left(X_{2}, h_{2}\right) \cap \bar{Z} \text {. }
$$

From (64), we obtain that if $\bar{Z}$ is nonempty then there is a $P_{0}$ which is in $Z \cap D\left(X_{2}, h_{2}\right)$. We shall show that no such $P_{0}$ exists.

First, suppose $P_{0}$ is an isolated point of $Z$. Then using the fact that an harmonic function is equal to its average, we obtain from (63) that for $X$ close to $P_{0}$ the following fact obtains:

$$
\tilde{f}(X)-\tilde{f}\left(P_{0}\right)=\tilde{f}_{\left|X-P_{0}\right|}(X)-\tilde{f}_{\left|X-P_{0}\right|}\left(P_{0}\right)+\tilde{f}_{\left|X-P_{0}\right|}\left(P_{0}\right)-\hat{f}\left(P_{0}\right) .
$$

We observe from (54) that $f$ is continuous in a neighborhood of $P_{0}$. We consequently obtain from (52), Lemma 2 , (66), and (67) that $\lim _{X \rightarrow P_{0}} \tilde{f}(X)=\tilde{f}\left(P_{0}\right)$. But then $\tilde{f}$ is continuous at $P_{0}$ and therefore $P_{0}$ is not in $Z$. Consequently, if $\bar{Z}$ is nonempty, there must be a point $P_{0}$ in $Z \cap D\left(X_{2}, h_{2}\right)$ which is a nonisolated point of $Z$. We shall show that this fact also leads to a contradiction.

Let $\varepsilon>0$ be given. Then it follows from (66) that we can choose $D\left(P_{0}, 4 h_{3}\right)$ with $h_{3}>0$ such that

$$
\left|\tilde{f}(X)-\tilde{f}\left(P_{0}\right)\right|<\varepsilon, \quad\left|\tilde{f}_{h}(X)-\tilde{f}(X)\right|<\varepsilon, \quad \text { and } \quad\left|\tilde{f}_{h}(X)-\tilde{f}_{h}\left(P_{0}\right)\right|<\varepsilon
$$

for $X$ in $D\left(P_{0}, 4 h_{3}\right) \cap \bar{Z}$ and $0<h<h_{3}$ and such that

$$
\bar{D}\left(P_{0}, 4 h_{3}\right) \subset \mathscr{S} \text { and } \bar{D}\left(P_{0}, 4 h_{3}\right) \subset D\left(X_{2}, h_{2}\right) \text {. }
$$


It follows from (52), (54), (69), and Lemma 2 that there exists $h_{4}$ with $0<h_{4}<h_{3}$ such that

$$
\begin{aligned}
& \text { if } X \text { and } X^{\prime} \text { are in } D\left(P_{0}, h_{3}\right) \text { and if } \\
& \left|X-X^{\prime}\right| \leqq h_{4} \text { then }\left|\tilde{f}_{\left|X-X^{\prime}\right|}\left(X^{\prime}\right)-\tilde{f}_{\left|X-X^{\prime}\right|}(X)\right|<\varepsilon .
\end{aligned}
$$

Now let $X$ be in $D\left(P_{0}, h_{4} / 2\right)$ and suppose $X$ is not in $\bar{Z}$. Then there exists a point $X^{\prime}$ in $\bar{Z}$ such that $\left|X-X^{\prime}\right|$ minimizes the distance of $X$ to $\bar{Z}$. Since $D\left(X,\left|X-X^{\prime}\right|\right)$ $\subset D\left(P_{0}, 4 h_{3}\right)$ we obtain that $f$ is continuous in $D\left(X,\left|X-X^{\prime}\right|\right)$ and therefore from (63) that $\tilde{f}_{\left|X-X^{\prime}\right|}(X)=\tilde{f}(X)$. But $\left|X-X^{\prime}\right|<h_{4} / 2$ and $X$ and $X^{\prime}$ are in $D\left(P_{0}, h_{3}\right)$. Therefore

$$
\begin{aligned}
\left|\tilde{f}(X)-\tilde{f}\left(P_{0}\right)\right| \leqq & \left|\tilde{f}_{\left|X-X^{\prime}\right|}(X)-\tilde{f}_{\left|X-X^{\prime}\right|}\left(X^{\prime}\right)\right| \\
& +\left|\tilde{f}_{\left|X-X^{\prime}\right|}\left(X^{\prime}\right)-\tilde{f}\left(X^{\prime}\right)\right|+\left|\tilde{f}\left(X^{\prime}\right)-\tilde{f}\left(P_{0}\right)\right|,
\end{aligned}
$$

and we conclude consequently from (68) and (70) that

$$
\left|\tilde{f}(X)-\tilde{f}\left(P_{0}\right)\right|<3 \varepsilon \text { for } X \text { in } D\left(P_{0}, h_{4} / 2\right) \cap \bar{Z}^{c},
$$

where $\bar{Z}^{c}$ represents the complement of $\bar{Z}$.

But from (68) we obtain that the inequality in (71) also holds for $X$ in $D\left(P_{0}, h_{4} / 2\right) \cap \bar{Z}$. Consequently $\tilde{f}$ is continuous at $P_{0}$. Therefore $P_{0}$ is not in $Z$, and we have arrived at a contradiction. We conclude that $\bar{Z}$ is empty and therefore that $Z$ is empty, and (57) is therefore established.

Next, we invoke (63) and conclude from (57) that

$$
f(X) \text { is harmonic in } \mathscr{S} \text {. }
$$

Now, $f(X)$ is periodic of period $2 \pi$ in the $x$-variable; so with $t>0$ on setting

$$
W^{\prime}(X, t)=-\sum f(M)\left(m^{2}-n^{2}\right) \exp \left(i(M, X)-|M|^{2} t\right),
$$

we obtain from (72), Lemma 6 , and the fact that the Fourier series of $f$ is given by (55) that for $0<\varepsilon<\pi$

$$
\lim _{t \rightarrow 0} W^{\prime}(X, t)=0 \text { uniformly for }-\infty<x<\infty \text { and }|y| \leqq(\pi-\varepsilon) .
$$

We next propose to show

$$
m_{0}^{2} \neq n_{0}^{2} \text { implies } \hat{f}\left(M_{0}\right)=0 .
$$

To establish (75), we observe from (74) that

$$
\lim _{t \rightarrow 0} \int_{-\pi}^{\pi} e^{i m_{0} x} e^{m_{0}^{2} t} W^{\prime}(X, t) d x=0 \quad \text { uniformly for }|y| \leqq \pi-\varepsilon .
$$

Therefore on setting

$$
b_{n}=\hat{f}\left(m_{0}, n\right)\left(m_{0}^{2}-n^{2}\right),
$$


we obtain from (76) that for $0<\varepsilon<\pi$,

$$
\lim _{t \rightarrow 0} g(y, t)=0 \quad \text { uniformly for }|y| \leqq \pi-\varepsilon,
$$

where

$$
g(y, t)=\sum_{n=-\infty}^{\infty} b_{n} \exp \left(\text { iny }-|n|^{2} t\right) .
$$

Next, we note from (50) that for $|y| \leqq \pi, f(x,-y)=-f(x, y)$. Consequently $\hat{f}\left(m_{0}, n\right)=-\hat{f}\left(m_{0},-n\right)$ for $n=0, \pm 1, \ldots$ Therefore,

$$
b_{0}=0 \text { and } b_{n}=-b_{-n} \text { for } n=1,2, \ldots
$$

We next set for $t>0$,

$$
G(y, t)=-\sum_{n=-\infty}^{\infty} b_{n} n^{-2} \exp \left(\text { iny }-|n|^{2} t\right)
$$

and observe from (79) and (80) that for $t>0$

$$
\partial^{2} G(y, t) / \partial y^{2}=\partial G(y, t) / \partial t=g(y, t) .
$$

It follows from (78) and (82) that

$$
\lim _{t \rightarrow 0} G(y, t)=G(y) \text { uniformly for }|y| \leqq \pi-\varepsilon .
$$

From (83), it follows that

$$
G(y) \text { is continuous for }|y|<\pi .
$$

We shall show even more holds; namely,

$$
G(y) \text { is linear for }|y|<\pi .
$$

To establish (85), we observe from (84) and [7, p. 431] that we need only show for fixed $y_{0}$ with $\left|y_{0}\right|<\pi$ that

$$
\lim _{h \rightarrow 0}\left[G\left(y_{0}+h\right)+G\left(y_{0}-h\right)-2 G\left(y_{0}\right)\right] h^{-2}=0 .
$$

We establish (86) by observing from (82) and (83) that for $h$ positive and such that $\left[y_{0}-h, y_{0}+h\right]$ is contained in the interior of the interval $(-\pi, \pi)$

$$
\begin{aligned}
G\left(y_{0}+h\right)+G\left(y_{0}-h\right)-2 G\left(y_{0}\right) & =\lim _{t \rightarrow 0}\left[G\left(y_{0}+h, t\right)+G\left(y_{0}-h, t\right)-2 G\left(y_{0}, t\right)\right] \\
& =\lim _{t \rightarrow 0} \int_{0}^{h} d \xi \int_{-\xi}^{\xi} g\left(y_{0}+\eta, t\right) d \eta=0 .
\end{aligned}
$$

(86) follows immediately from this fact, and therefore (85) is established.

From (80), it follows that $G(0, t)=0$ for $t>0$, and therefore from (83) that $G(0)=0$. We consequently conclude from (85) that there is a constant $\beta$ such that

$$
G(y)=\beta y \text { for }-\pi<y<\pi .
$$


Next from (77), we see that

$$
b_{n} n^{-2}=o(1) \quad \text { as }|n| \rightarrow \infty .
$$

Since for a series with bounded terms Gaussian summability implies Abel summability (See [1, p. 39]. The theorem stated there is given in terms of summability for integrals, but the proof can be carried over to summability for series with no difficulty.), we conclude from (80), (81), (83), and (87) that

$$
\lim _{t \rightarrow 0} \sum_{n=1}^{\infty} 2 i b_{n} n^{-2} \sin n y e^{-|n| t}=-\beta y \text { for }-\pi<y<\pi .
$$

But then it follows from (88), (89), and [8, p. 362] that for $n=1,2, \ldots$

$$
2 i b_{n} n^{-2}=-\beta \int_{-\pi}^{\pi} y \sin n y d y / \pi=2 \beta(-1)^{n+1} n^{-1} .
$$

If $m_{0}=0$, (75) is immediate from (50). If $m_{0} \neq 0$, then (77) implies $b_{m_{0}}=0$ and consequently (80) and (90) imply $\beta=0$. But then $\beta=0$ and (90) imply $b_{n}=0$ for $n=1,2, \ldots$ Therefore (77) and (80) imply that

$$
\hat{f}\left(m_{0}, n_{0}\right)\left(m_{0}^{2}-n_{0}^{2}\right)=0 .
$$

But (91) implies (75), and (75) is therefore established.

We conclude from (50) and (75) that

$$
\hat{f}(m, n)=0 \text { for } m=0 \text { or } n=0 \text { or } m^{2} \neq n^{2},
$$

and

$$
\hat{f}(m, m)=\hat{f}(-m,-m)=-\hat{f}(-m, m)=-\hat{f}(m,-m) \text { for } m \neq 0 .
$$

Next we define the set $Q^{\prime}$ as follows:

$$
Q^{\prime}=\left\{x:-\pi \leqq x \leqq \pi \text { and } \limsup _{y \rightarrow 0+}|[f(x, y)-f(x,-y)] / y| \neq 0\right\} .
$$

It follows from assumption (v) of Theorem 2 and from (50) that

$$
Q^{\prime} \text { is a countable set contained in }(-\pi, \pi) \text {. }
$$

Now for each fixed $x$, it follows from (51) and (54) that $f(x, y)$ is a bounded continuous function of $y$ for $-\pi<y<\pi$. Consequently, $f(x, y)$ can be expanded in a Fourier series with Fourier coefficients $\alpha_{m}(x)$, that is

$$
f(x, y) \sim \sum_{m=-\infty}^{\infty} \alpha_{m}(x) e^{i m y} .
$$

Also, from (51), (54), (92), and the periodicity of $f$, we obtain that $\alpha_{m}(x)$ is a periodic function of $x$ and in particular that for $-\pi \leqq x \leqq \pi$

$$
\alpha_{m}(x)=\hat{f}(m, m) e^{i m x}+\hat{f}(-m, m) e^{-i m x} \quad m= \pm 1, \pm 2, \ldots
$$

and

$$
\alpha_{0}(x)=0
$$


Using Fatou's theorem and (94), we next obtain that

$$
\lim _{t \rightarrow 0+} \sum_{m=-\infty}^{\infty} i m \alpha_{m}(x) e^{-|m| t}=0 \text { for } x \text { in }[-\pi, \pi]-Q^{\prime} .
$$

From (93), (97), and (98), it follows that $m \alpha_{m}(x)=-m \alpha_{-m}(x)$ for $m=0,1,2, \ldots$ Consequently, using (93) once again, it follows from (99) that

$$
\lim _{t \rightarrow 0+} \sum_{m=-\infty}^{\infty} \operatorname{im} \hat{f}(m, m) e^{i m x} e^{-|m| t}=0 \text { for } x \text { in }[-\pi, \pi]-Q^{\prime} .
$$

Next we define $f_{2}(X)$ as in Lemma 4, and obtain from (54) and Lemma 4 that

$$
f_{2}(x, 0) \text { is uniformly smooth in } x \text { for }-\infty<x<\infty \text {. }
$$

Also, we observe that

$$
\begin{aligned}
\hat{f}_{2}(M) & =\inf (M) /|M|^{2} & & \text { for } M \neq 0 \\
& =0 & & \text { for } M=0 .
\end{aligned}
$$

Since $f_{2}(x, y)$ is a continuous periodic function of period $2 \pi$ in each variable, we can expand $f_{2}(x, 0)$ in a Fourier series with Fourier coefficients $\gamma_{m}$ and obtain from (92), (93), and (102) that

$$
f_{2}(x, 0) \sim \sum_{m=-\infty}^{\infty} \gamma_{m} e^{i m x}
$$

where

$$
\begin{aligned}
\gamma_{m} & =i m \hat{f}(m, m) / m^{2} & & \text { for } m= \pm 1, \pm 2, \ldots \\
& =0 & & \text { for } m=0 .
\end{aligned}
$$

Next, we let $\bar{D}^{2} f_{2}(x, 0)$ and $\underline{D}^{2} f_{2}(x, 0)$ represent respectively the upper and lower second symmetric derivatives of $f_{2}(x, 0)$. (See [8, p. 319].) It follows from [8, p. 353] and from (100) and (103) that

$$
\bar{D}^{2} f_{2}(x, 0) \geqq 0 \text { and } \underline{D}^{2} f_{2}(x, 0) \leqq 0 \text { for } x \text { in }[-\pi, \pi]-Q^{\prime} .
$$

Next, it follows from (95), (101), and (104) and from [8, p. 328] and the fact that $f_{2}(x, 0)$ is a continuous function of $x$ that

$$
f_{2}(x, 0) \text { is identically a constant for }-\pi<x<\pi \text {. }
$$

But then (103) and (105) imply that

$$
\hat{f}(m, m)=0 \text { for } m= \pm 1, \pm 2, \ldots
$$

We conclude from (92), (93), and (106) that

$$
\hat{f}(M)=0 \text { for all } M .
$$

From (50), (54), and (107), we then obtain that $f(X)=0$ for all $X$ and therefore that $u(X)=0$ for $X$ in $S^{\pi}$. The proof to the theorem is complete. 
5. Appendix. Letting $C_{c}^{\infty}\left[D\left(X^{*}, R_{0}\right)\right]$ represent the class of functions in class $C^{\infty}$ on the open disc $D\left(X^{*}, R_{0}\right)$ having compact support within this disc, we say that a function $u$ locally in $L^{1}$ on $D\left(X^{*}, R_{0}\right)$ has a weak wave equal to zero in $D\left(X^{*}, R_{0}\right)$ provided

$$
\int_{D\left(X^{*}, R_{0}\right)} u(X)\left[\lambda_{x x}(X)-\lambda_{y y}(X)\right] d X=0 \text { for } \lambda \text { in } C_{c}^{\infty}\left[D\left(X^{*}, R_{0}\right)\right]
$$

With $\square u$ representing the generalized wave of $u$ defined in $\S 1$, the first remark that we establish is the following:

REMARK 1. If $u$ is locally in $L^{1}$ on $D\left(X^{*}, R_{0}\right)$ and the weak wave of $u$ is zero in $D\left(X^{*}, R_{0}\right)$, then $\square u(X)=0$ for all $X$ in $D\left(X^{*}, R_{0}\right)$.

Remark 1 is an immediate consequence of the following three elementary statements:

Statement 1. Let $u$ be in $C^{2}\left[D\left(X_{0}, r_{0}\right)\right]$ and suppose $u_{x x}(X)-u_{y y}(X)=0$ for $X$ in $D\left(X_{0}, r_{0}\right)$. Then

$$
\int_{D(0, r)} u\left(X_{0}+X\right)\left(x^{2}-y^{2}\right) d X=0 \text { for } 0<r<r_{0}
$$

STATEMENT 2. Let $\left\{u_{n}\right\}_{n=1}^{\infty}$ be a sequence of functions in $C^{2}\left[D\left(X^{*}, R_{0}\right)\right]$ having the property that for every $n, u_{n x x}(X)-u_{n y y}(X)=0$ for $X$ in $D\left(X^{*}, R_{0}\right)$. Suppose also that $u$ is locally in $L^{1}$ on $D\left(X^{*}, R_{0}\right)$ and that

$$
\lim _{n \rightarrow \infty} \int_{D\left(X^{*}, R\right)}\left|u_{n}(X)-u(X)\right| d X=0 \text { for } 0<R<R_{0} .
$$

Then if $\bar{D}\left(X_{0}, r\right) \subset D\left(X^{*}, R_{0}\right)$, the following fact holds:

$$
\int_{D(0, r)} u\left(X_{0}+X\right)\left(x^{2}-y^{2}\right) d X=0
$$

STATEMENT 3. Suppose $u$ is locally in $L^{1}$ on $D\left(X^{*}, R_{0}\right)$ and the weak wave of $u$ is zero in $D\left(X^{*}, R_{0}\right)$. Then if $\bar{D}\left(X_{0}, r\right) \subset D\left(X^{*}, R_{0}\right)$ the following fact holds:

$$
\int_{D(0, r)} u\left(X_{0}+X\right)\left(x^{2}-y^{2}\right) d X=0
$$

Next, we show that the converse of Remark 1 is false. In particular we shall establish the following remark.

REMARK 2. There exists a function $u$ in $L^{1}[D(0,1)]$ such that $\square u(X)=0$ for $X$ in $D(0,1)$ and such that $u$ does not have the following property: the weak wave of $u$ is zero in $D(0,1)$.

We define $u(X)$ as follows: $u(X)=1$ for $y>0, u(X)=\frac{1}{2}$ for $y=0, u(X)=0$ for $y<0 . u$ is clearly in $L^{1}[D(0,1)]$, and since $\int_{0}^{\pi} \cos 2 \theta d \theta=0, \square u(X)=0$ for $X$ in 
$D(0,1)$. To show that the statement "the weak wave of $u$ is zero in $D(0,1)$ " is false we observe from Statement 3 above that we need only show

$$
\int_{D(0,1 / 2)} u\left(x,-\frac{1}{4}+y\right)\left(x^{2}-y^{2}\right) d x d y \neq 0 .
$$

An easy computation shows that the integral in (108) is equal to $-3^{1 / 2} / 2^{7}$, and Remark 2 is established.

\section{REFERENCES}

1. S. Bochner and K. Chandrasekharan, Fourier transforms, Princeton Univ. Press, Princeton, N. J., 1949.

2. A. P. Calderón and A. Zygmund, Singular integrals and periodic functions, Studia Math. 14 (1954), 249-271.

3. F. Hausdorff, Set theory, Chelsea, New York, 1957.

4. S. Saks, Theory of the integral, English transl. Monogr. Mat. Vol. II, Vol. VII, PWN, Warsaw, 1937.

5. V. L. Shapiro, Fourier series in several variables, Bull. Amer. Math. Soc. 70 (1964), 48-93.

6. - The divergence theorem for discontinuous vector fields, Ann. of Math. 68 (1958), 604-624.

7. E. C. Titchmarsh, The theory of functions, Oxford Univ. Press, London, 1952.

8. A. Zygmund, Trigonometric series, Vol. 1, Cambridge Univ. Press, New York, 1959.

UNIVERSITY OF CALIFORNIA,

Riverside, CALIfornia 